\title{
Network analysis of gene fusions in human cancer
}

\author{
Morgan Harrell ${ }^{1}$, Junfeng Xia ${ }^{1}$, Zhongming Zhao ${ }^{1,2,3^{*}}$ \\ From 12th Annual UT-ORNL-KBRIN Bioinformatics Summit 2013 \\ Buchanan, TN, USA. 22-24 March 2013
}

\section{Background}

Gene fusions are hybrid genes formed when two discrete genes are incorrectly joined together. Gene fusions are found to play roles in tumorigenesis. For example, the fusion gene $B C R-A B L$ translates into an abnormal tyrosine kinase that accelerates development of chronic myelogenous leukemia [1]. A network is a relational representation of nodes (e.g., genes) with edges, and is a useful approach to explore biological interactions among many related nodes. Network analysis of gene fusions in cancer would aid the exploration of gene fusion occurrence and association with tumorigenesis. Hoglund et al [2] performed an initial investigation of gene fusions network after collecting 291 tumorigenesis related gene fusions from the Mitelman database in 2006. Since then, gene fusion data has exponentially increased. There is no current and comprehensive cancer-related gene fusion network to assist in targeting cancer-associated genes.

\section{Materials and methods}

We mined three public databases for cancer-related gene fusion sequences, and one database for fusions records from cancer studies, transcriptome analysis, and genetic disorders. Specifically, we processed each dataset by removing incomplete entries and then extracted gene-fusion pairs. Genes serve as the nodes in the network and each fusion pair is joined by an edge. Repeating pairs were represented once. We used Cytoscape to build five networks: one for each dataset and the fifth that encompasses all datasets. We graphed the occurrence of degree in each single dataset network to determine an empirical definition for hub genes.

\footnotetext{
* Correspondence: zhongming.zhao@vanderbilt.edu

'Department of Biomedical Informatics, Vanderbilt University School of Medicine, Nashville, TN 37203, USA

Full list of author information is available at the end of the article
}

\section{Results}

The comprehensive network includes 9852 genes, displays 12,791 relationships, and highlights 1248 hub genes. The network highlights genes such as MLL and MALAT1, both of which have roles in tumorigenesis. The network also highlights genes such as WDR74 and COL1A1, which are not much studied.

\section{Conclusions}

This preliminary network analysis provides interesting features of tumorigenesis-related fusions. Further systematic analysis of gene fusion networks may aid researchers to better understand cancer gene fusions and test novel fusions in specific types of cancer.

\section{Acknowledgements \\ We would like to thank the members in Bioinformatics and Systems Medicine Laboratory for their valuable discussion in this project. This work was partially supported by the National Library of Medicine Training Grant 2T15LM007450-11 and the Stand Up To Cancer-American Association for Cancer Research Innovative Research Grant (SU2C-AACR-IRG0109) and the VICC Cancer Center Core grant P30CA68485 from National Institutes of Health.}

\section{Authors' details}

'Department of Biomedical Informatics, Vanderbilt University School of Medicine, Nashville, TN 37203, USA. 'Department of Psychiatry, Vanderbilt University School of Medicine, Nashville, TN 37232, USA. ${ }^{3}$ Department of Cancer Biology, Vanderbilt University Medical Center, Nashville, TN 37232, USA.

Published: 22 October 2013

\section{References}

1. Bartram $C R$, de Klein $A$, Hagemeijer $A$, van Agthoven $T$, Geurts van Kessel $A$, Bootsma D, Grosveld G, Ferguson-Smith MA, Davies T, Stone M, et al: Translocation of $\mathrm{c}-\mathrm{ab} 1$ oncogene correlates with the presence of a Philadelphia chromosome in chronic myelocytic leukaemia. Nature 1983, 306:277-280.

2. Hoglund M, Frigyesi A, Mitelman F: A gene fusion network in human neoplasia. Oncogene 2006, 25:2674-2678.

doi:10.1186/1471-2105-14-S17-A13

Cite this article as: Harrell et al:: Network analysis of gene fusions in human cancer. BMC Bioinformatics 2013 14(Suppl 17):A13.

\section{(Ciomed Central}

